\title{
Highly Accurate Energy-Conserving Flexible Touch Sensors
}

\author{
Huimin Lu, ${ }^{*}$ Yujie Li, ${ }^{1 * *}$ Yun Li, ${ }^{1}$ Seiichi Serikawa, and Hyoungseop Kim \\ Kyushu Institute of Technology, Sensui-cho 1-1, Tobata-ku, Kitakyushu, Fukuoka 804-8550, Japan \\ ${ }^{1}$ Yangzhou University, Huayang West Rd., Yangzhou 225127, China
}

(Received September 6, 2016; accepted October 27, 2016)

Keywords: touch sensor, Internet of Things, energy-conserving device, flexible sensor

Smart touch sensors are used in many applications, such as the iPhone and some smart home systems. Recent touch sensors perform well in consumer electrical devices; however, there are some drawbacks. For example, most touch sensors have low accuracy for detecting human movements. Many require significant power and have a fixed shape. We propose a flexible, highly accurate, and energy-conserving touch sensor. Our primary contributions are as follows: (1) an energy-conserving touch sensor is developed and tested experimentally; (2) a flexible and arbitrarily shaped touch sensor is designed; and (3) the manufacturing cost is very low and up to 200 touch sensors can be connected to the system. As a result, this energy-conserving touch sensor can be fabricated using common manufacturing processes for consumer electronic devices.

\section{Introduction}

Switches are widely used in modern electronic devices. Typically, traditional mechanical switches have two contacts that must be connected to complete an electrical circuit. Most mechanical switches produce noise. In addition, they can be unreliable, have a high failure rate, and are subject to wear. Thus, they cannot satisfy the requirements of modern systems. ${ }^{(1)}$

Various types of smart switches, such as voice-operated, ${ }^{(2)}$ optoelectronic, ${ }^{(3-5)}$ touch, ${ }^{(6)}$ and pressure switches, ${ }^{(7)}$ have been developed to replace mechanical switches. Such switches have more functions than normal mechanical switches; however, they still have some limitations. For example, with voice-operated switches, the voice-recognition function may not perform well in a noisy environment. Optoelectronic switches are reliable; however, they require a relatively high driving voltage, which limits switching speed. A mechanical pressure switch does not require a power supply and is simple and convenient to use. However, the pressure switch needs mechanical movement due to mechanical deformation. Consequently, accuracy is low. Typically, touch switches are small, lightweight devices with a fixed shaped. In this paper, we describe a highly accurate energy-conserving flexible touch sensor.

\footnotetext{
*Corresponding author: e-mail: luhuimin@ieee.org

${ }^{* *}$ Corresponding author: e-mail: liyujie@yzu.edu.cn

http://dx.doi.org/10.18494/SAM.2017.1458
} 


\section{Materials and Methods}

Touch switch sensors can be divided into three parts, i.e., a touch switch unit, a signal processing unit, and a detection unit. Figure 1 shows a block diagram of a touch switch sensor. Each touch sensor $S_{i}(i \in\{1, \ldots, n\})$ has a subcircuit and relay switch as the signal processing unit to determine the input signal. $n$ is the number of touch switches. The detection unit differentiates sensor signals. In a system with many switches, the detection unit must be able to determine which switch has been touched. In the proposed system, an LED light $L_{i}(i \in\{1, \ldots, n\})$ is used to show the effectiveness of the equipment. We describe the details of the proposed touch switch, subcircuit, relay, and detection units in the following.

Aluminum foil conductive adhesive tape \{thickness, $0.05 \mathrm{~mm}$; peel adhesion, 12.7 (1295) [N (gf)/W25 mm]; electrical resistance, $0.06 \Omega / \mathrm{cm}^{2}$; Teraoka Seisakusho Co. Ltd. $\left.{ }^{(8)}\right\}$ with excellent depthwise and lengthwise conductivity is used in this system (Fig. 2). Under normal environmental conditions, the tape is waterproof, dustproof, and stretch resistant and demonstrates good stability and robustness, as well as softness and conductivity. We have designed switch panels of various shapes (Fig. 3).

In subcircuits (Fig. 4), the touch panel connects to two resistors connected in series. Input to the resistors is a clock signal, a high-frequency pulse width modulation (PWM) wave (voltage, 5 $\mathrm{V}$; frequency, $10 \mathrm{kHz}$ ). The average voltage between the resistors is the input to a buffer circuit. When the switch panel is open, the middle voltage is one-half the input voltage. After the buffer and rectifier circuit, the input to the voltage comparator is almost a fixed value. If the inverting input potential of the comparator is less than the threshold value, then the output of the comparator is low and the metal-oxide-semiconductor field-effect transistor $\left(\right.$ MOSFET) ${ }^{(9)}$ is off, i.e., the switch is closed.

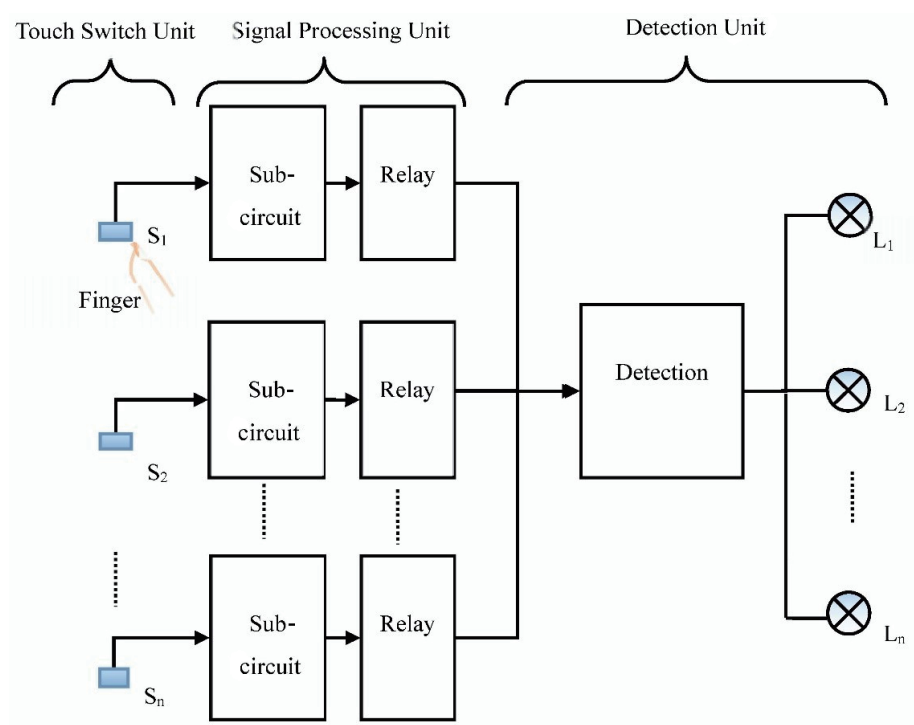

Fig. 1. (Color online) Structure of touch sensor system. 


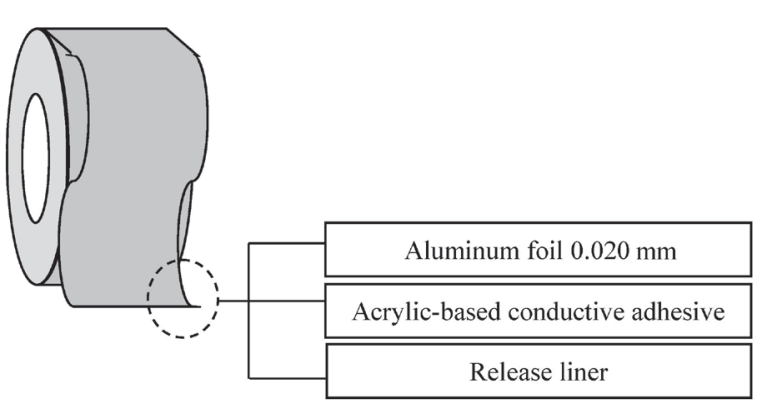

Fig. 2. Structure of conductive adhesive tape.

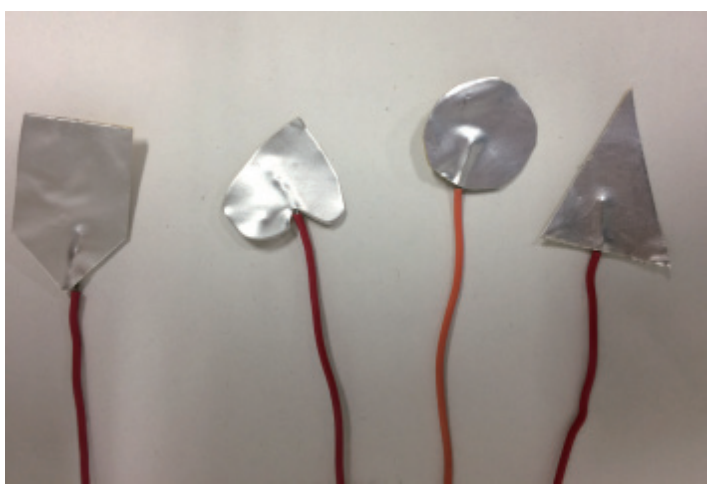

Fig. 3. (Color online) Switch panels.

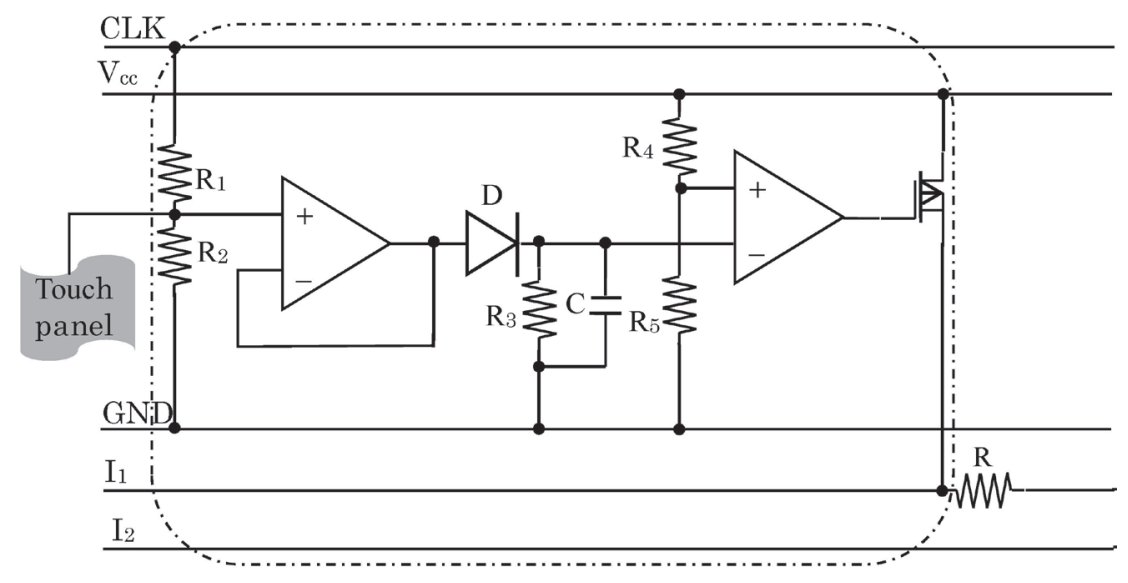

Fig. 4. Subcircuit of signal processing unit.

When a finger touches a switch panel, one branch of the alternating current flows through the panel and the human body to the ground. To reduce the impact on the human body, the resistance is very high, i.e., $R_{1}=R_{2}=4 \mathrm{M} \Omega$. The human body has high impedance to alternating current. The current is under order of $0-1 \mu \mathrm{A}$, which is very low compared with conventional touch switch methods. Thus, when a human touches the switch panel, the total impedance is increased and the voltage input to the buffer circuit is decreased. After the buffer and rectifier circuits, it becomes a fixed value. To insure that the value is higher than the potential of the inverting input of the comparator, the output of comparator should be high, and then the MOSFET should be on, i.e., the switch should be open. After testing, $R_{4}=3 \mathrm{k} \Omega$ and $R_{5}=2 \mathrm{k} \Omega$. The voltage $V_{c c}$ affects the resistance $R$ through the MOSFET. As the internal resistance of the MOSFET is very small, we consider that the voltage power supply directly affects resistance.

In the signal processing unit, a relay switch is used to control the connection between subcircuits and the detection unit. An $N$-channel MOSFET is used in this circuit. It has low resistance, very high speed switching, and low-voltage drive.

In Fig. 5, each switch comprises a signal processing unit. The resistors are connected to each other and to a detection unit. The detection unit is shown by the dashed line in Fig. 5, and the right 


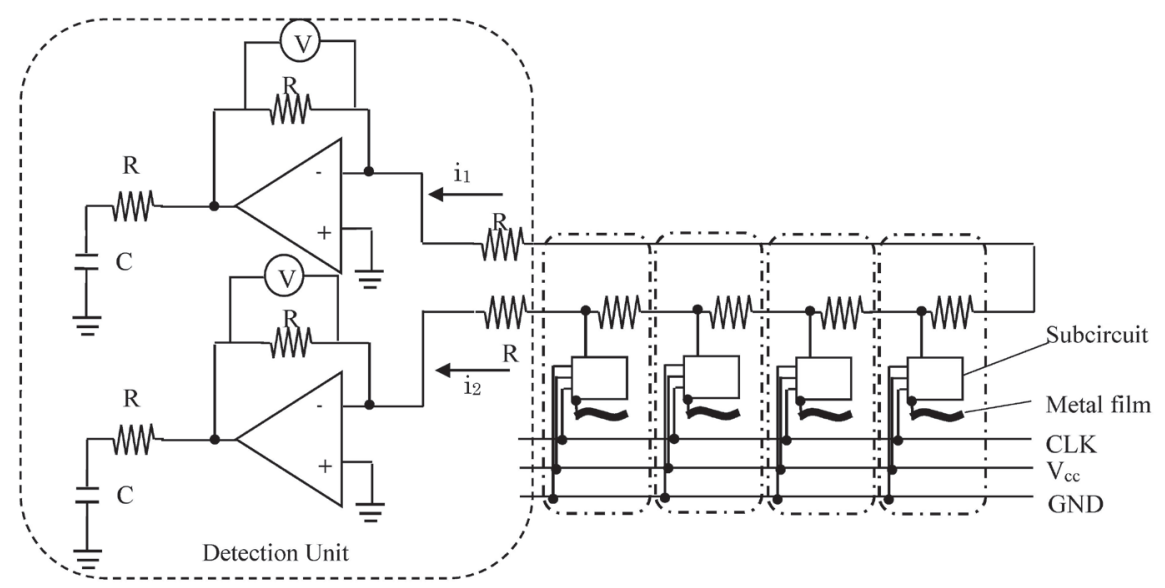

Fig. 5. Circuit of detection unit.

part of the figure is the switch. Each switch is connected through a resistance to the I/V converter circuit. If a metal film is touched, then the switch is turned on and the resistance directly connects to the voltage power supply. There are two currents, i.e., $i_{1}$ and $i_{2}$, that flow in two directions into the I/V converter circuits. If the feedback resistance voltage in the converter circuit can be measured, the current flowing through resistances can be calculated. To simplify this calculation, the feedback circuit is made equal to the input resistance of the amplifier. This relationship is expressed as follows.

$$
\begin{gathered}
i=-\frac{V}{R} \\
\left\{\begin{array}{l}
i_{1}=-\frac{V_{1}}{R} \\
i_{2}=-\frac{V_{2}}{R}
\end{array}\right. \\
i_{1}=\frac{V_{c c}}{(n+1) R} \\
i_{2}=\frac{V_{c c}}{(N+1-n) R}
\end{gathered}
$$

Rate is set as the ratio of the currents $i_{1}$ and $i_{2}$, which is expressed as follows.

$$
\text { Rate }=\frac{i_{2}-i_{1}}{i_{2}+i_{1}}
$$

By substituting Eq. (2) into Eq. (5), we obtain the following:

$$
\text { Rate }=\frac{i_{2}-i_{1}}{i_{2}+i_{1}}=\frac{2 n-N}{n+2}
$$


where $V_{1}$ and $V_{2}$ are the voltages of two resistances in two branches. Assuming that the total number of switch units in various shapes is $N$ and the touched switch number is $n$, the DC power voltage is $V_{c c}$. Equation (6) is a theoretical expression to determine the switch position. Once the voltages $V_{1}$ and $V_{2}$ are known, the real and ideal rates can be compared to judge the switch ID.

\section{Experimental Results and Discussion}

In this paper, we have proposed a touch sensor system. The feasibility of this principle has been verified through simulation and real-world experiments. We used the switch units in various shapes.

The model is based on the switch system shown in Fig. 6. The circuit of the switch is shown in Fig. 7. All connected resistances in the switch have the same value, i.e., $1 \mathrm{k} \Omega$. Here, the clock signal is a PWM wave of $10 \mathrm{kHz}$ frequency, which is produced by a PSoC chip. The $V_{c c}$ is $5 \mathrm{~V}$ and the GND is produced by PSoC MiniProg. The detection of the switch number requires two steps. First, the current is detected, and then the result is calculated to determine the position. The first part is realized in the broad circuit, and the second part is realized by programming the microcomputer chip.

Theoretically, the touch switch ID detection is based on Eq. (6). However, in real circuits, we generally calculate the rate as follows.

$$
\text { Rate }=\frac{I_{2}-I_{1}}{I_{2}+I_{1}}=\frac{V_{2}-V_{1}}{V_{2}+V_{1}}
$$

We compared the test rate to the theoretical rate in each experiment (Tables 1 to 4 ).

From these experiments, we conclude that the rate and switch ID demonstrate a nearly linear relationship. The largest rate error of each point is 0.0104 . The range of the rates is -1 to 1 . Thus, we can estimate the maximum number of switches as (range of rate)/(maximum error). The value is [1$(-1)] / 0.0104 \approx 200$. Thus, we can connect approximately 200 touch switch sensors in this system.

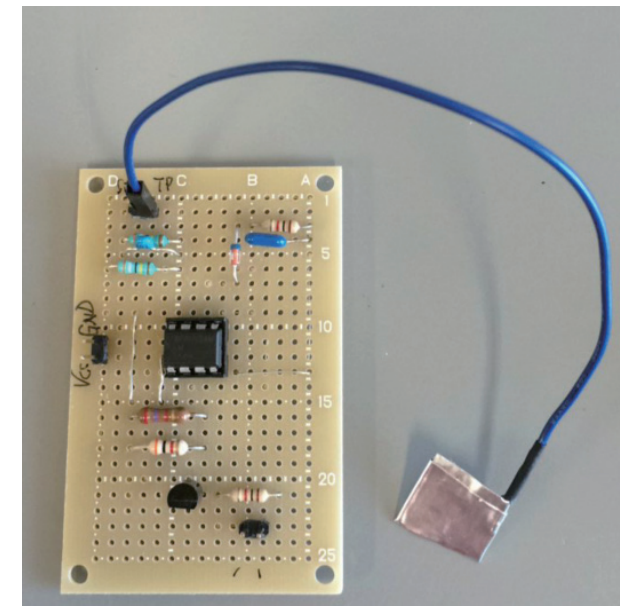

Fig. 6. (Color online) Touch switch and signal processing units.

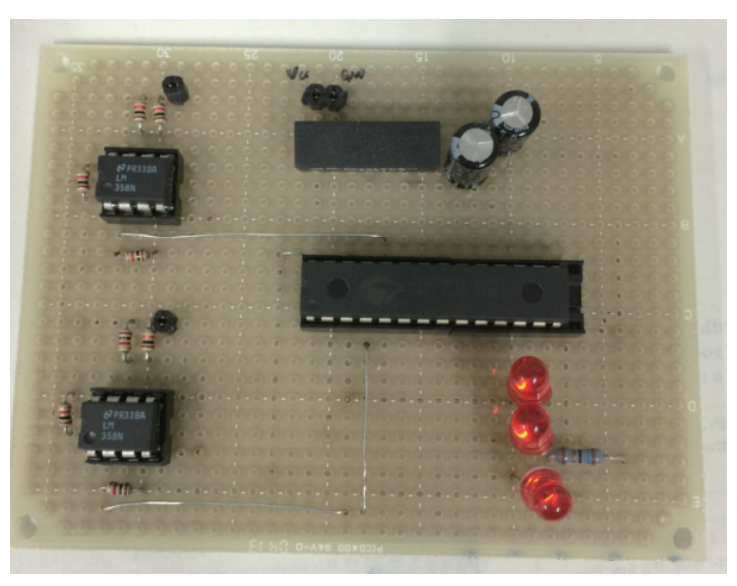

Fig. 7. (Color online) Detection unit. 
Table 1

Three switch sensors.

\begin{tabular}{lccc}
\hline Switch ID & Rate & Ideal rate & $\Delta R$ \\
\hline 1 & -0.605 & -0.6 & 0.0057 \\
2 & -0.195 & -0.2 & 0.0077 \\
4 & 0.206 & 0.2 & 0.0075 \\
\hline
\end{tabular}

Table 3

Five switch sensors.

\begin{tabular}{llcc}
\hline Switch ID & Rate & Ideal rate & $\Delta R$ \\
\hline 1 & -0.717 & -0.714 & 0.0080 \\
2 & -0.427 & -0.429 & 0.0103 \\
3 & -0.136 & -0.143 & 0.0093 \\
4 & 0.15 & 0.143 & 0.0101 \\
5 & 0.435 & 0.428 & 0.0102 \\
\hline
\end{tabular}

Table 2

Four switch sensors.

\begin{tabular}{lccc}
\hline Switch ID & Rate & Ideal rate & $\Delta R$ \\
\hline 1 & -0.679 & -0.667 & 0.0062 \\
2 & -0.329 & -0.33 & 0.0078 \\
3 & -0.004 & 0.0 & 0.0102 \\
4 & 0.342 & 0.33 & 0.0082 \\
\hline
\end{tabular}

Table 4

Six switch sensors.

\begin{tabular}{lccc}
\hline Switch ID & Rate & Ideal rate & $\Delta R$ \\
\hline 1 & -0.752 & -0.75 & 0.0047 \\
2 & -0.493 & -0.5 & 0.0099 \\
3 & -0.232 & -0.25 & 0.0101 \\
4 & 0.007 & 0 & 0.0104 \\
5 & 0.27 & 0.25 & 0.0094 \\
6 & 0.508 & 0.5 & 0.0085 \\
\hline
\end{tabular}

\section{Conclusions}

In this paper, we have proposed a highly accurate touch sensor system. After verifying the feasibility of the touch switch by simulation, a real circuit was built. Different shapes of touch panels were designed in this experiment, and the results show that the performance of the switch is suitable for practical applications. In the future, we will develop a software program that will improve the detection rate.

\section{Acknowledgments}

This work was supported by Japan Society for the Promotion of Science (JSPS) KAKENHI (No. 17K14694), Leading Initiative for Excellent Young Researchers (LEADER) of the Ministry of Education, Culture, Sports, Science and Technology, Japan (No. 16809746), Research Fund of Chinese Academy of Sciences (No. MGE2015KG02), Research Fund of the State Key Laboratory of Marine Geology in Tongji University (No. MGK1608), and Research Fund of the State Key Laboratory of Ocean Engineering in Shanghai Jiaotong University (Nos. 1315 and 1510).

\section{References}

1 Touch switch: https://en.wikipedia.org/wiki/Touch switch (accessed May 20, 2016).

2 T. Oka and K. Matsushima: Proc. 11th ACM/IEEE Int. Conf. Human-Robot Interaction (2016) pp. 487-488.

3 H. Lu and S. Serikawa: IEEJ Trans. Electr. Electron. Eng. 8 (2013) S110.

4 Y. Zhang, S. P. Bradley, and Z. Dong: Sensors 13 (2013) 4029.

5 S. Wan, Y. Zhang, and J. Chen: IEEE Sens. J. 16 (2016) 7433.

6 S. Serikawa and L. F. Zhang: Proc. 2nd Int. Conf. ICICI-BME (2011) pp. 8-13.

7 J. Tony: US Patent No. 20160003191 (2016).

8 Teraoka Seisakusho Co., Ltd.: http://www.teraokatape.co.jp (accessed May 20, 2016).

9 Y. Huang: J. Hubei Inst. Educ. 24 (2007) 35. 


\section{About the Authors}

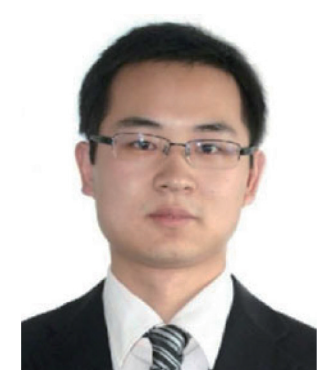

Huimin Lu received his B.S. degree in Electronics Information Science and Technology from Yangzhou University in 2008. He received his M.S. degree in Electrical Engineering from Kyushu Institute of Technology and Yangzhou University in 2011. He received his Ph.D. degree in Electrical Engineering from Kyushu Institute of Technology in 2014. From 2013 to 2016, he was a JSPS research fellow at Kyushu Institute of Technology. Currently, he is an Assistant Professor in Kyushu Institute of Technology. His research interests include computer vision, robotics, artificial intelligence, and ocean observation.

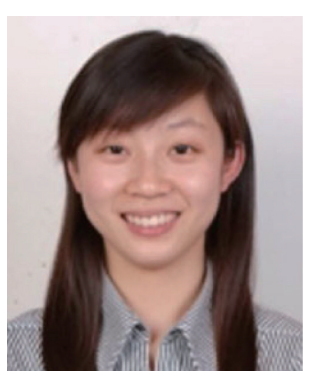

Yujie Li received her B.S. degree in Computer Science and Technology from Yangzhou University in 2009. She received her M.S. degree in Electrical Engineering from Kyushu Institute of Technology and Yangzhou University in 2012. She received her Ph.D. degree from Kyushu Institute of Technology in 2015. Currently, she is a Lecturer in Yangzhou University. Her research interests include computer vision, sensors, and image segmentation.

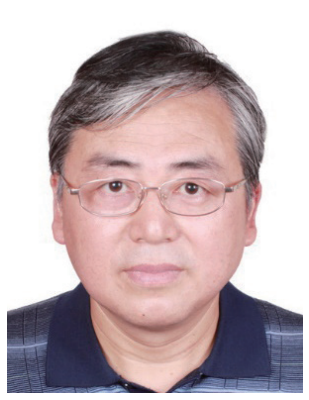

Yun Li received his M.S. degree in Computer Science and Technology from Hefei University of Technology in 1991 and his Ph.D. degree in Control Theory and Control Engineering from Shanghai University in 2005. He is a Professor in the School of Information Engineering, Yangzhou University, China. He is currently a Vice Dean of the School of Information Engineering of Yangzhou University. His research interests include pattern recognition, information fusion, data mining, and cloud computing.

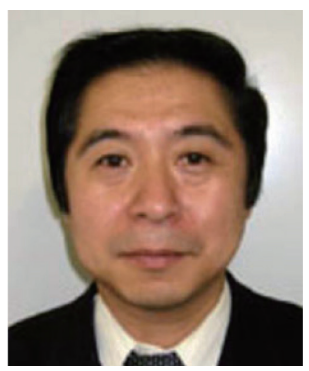

Seiichi Serikawa received his B.S. and M.S. degrees in Electronic Engineering from Kumamoto University in 1984 and 1986, respectively. He received his Ph.D. degree in Electronic Engineering from Kyushu Institute of Technology in 1994. Currently, he is a Vice President of Kyushu Institute of Technology and also serves as a Professor in the Center for Socio-Robotic Synthesis and the Department of Electrical and Electronic Engineering. His current research interests include computer vision, sensors, and robotics.

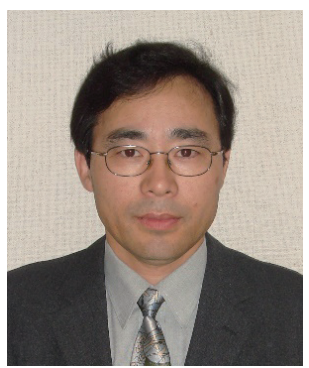

Hyoungseop Kim received his B.A. degree in Electrical Engineering from Kyushu Institute of Technology in 1994, and M.S. and Ph.D. degrees from Kyushu Institute of Technology in 1996 and 2001, respectively. He is a Professor in the Department of Control Engineering at Kyushu Institute of Technology. His research interests are focused on medical applications of image analysis. 